\title{
Flatter-Ulme (Ulmus laevis), Baum des Jahres 2019
}

\author{
Veit Martin Dörken \& Armin Jagel
}

\begin{abstract}
The fluttering elm (Ulmus laevis) is tree of the year 2019. It differs from the other two native elm species by their board roots. Due to river straightening and drainage of flood plains their stocks have declined. The fluttering elm is less affected by elm disease than the scots elm and the field elm. The elm disease is based on an infection by ascomycetes of the genus Ophiostoma. The fungi can invade the elm trees, if they have previously been attacked by the elm bark beetle (genus Scolytus).
\end{abstract}

\section{Zusammenfassung}

Die Flatter-Ulme (Ulmus laevis) ist Baum des Jahres 2019. Von den beiden anderen heimischen Ulmen-Arten unterscheidet sie sich deutlich durch ihre Brettwurzeln. Durch Flussbegradigungen und Trockenlegungen von Auengebieten sind ihre Bestände zurückgegangen. Die Flatter-Ulme ist seltener vom Ulmensterben betroffen als die Berg- und Feld-Ulme. Das Ulmensterben beruht auf einer Infektion durch Schlauchpilze der Gattung Ophiostoma. Die Pilze können in die Ulmen eindringen, wenn diese vorher vom Ulmensplintkäfer (Gattung Scolytus) befallen worden sind.

\section{Einleitung}

Wie in den letzten 30 Jahren kürte das Kuratorium Baum des Jahres auch für 2019 wieder eine heimische Baumart zum Baum des Jahres. In diesem Jahr konnte sich die Flatter-Ulme (Ulmus laevis) gegen die Konkurrenten Kornelkirsche und Douglasie durchsetzen. Mit der Wahl der Flatter-Ulme, die auch unter dem Namen Flatter-Rüster bekannt ist, soll diese weniger bekannte und recht selten gewordene Ulmen-Art einer breiteren Öffentlichkeit näher gebracht werden. Zudem steht sie stellvertretend für den Verlust ihres typischen Lebensraumes, der Fluss- und Bachauen inklusive der dortigen Pflanzengesellschaften. Von besonderem Interesse ist die Flatter-Ulme wegen ihrer wesentlich höheren Widerstandsfähigkeit gegenüber dem gefürchteten und weit verbreiteten Ulmensterben (s. u.). Dieser Krankheit sind bereits große Teile der Feld- und Berg-Ulmen-Bestände (U. minor und U. glabra) zum Opfer gefallen. In Nordrhein-West-

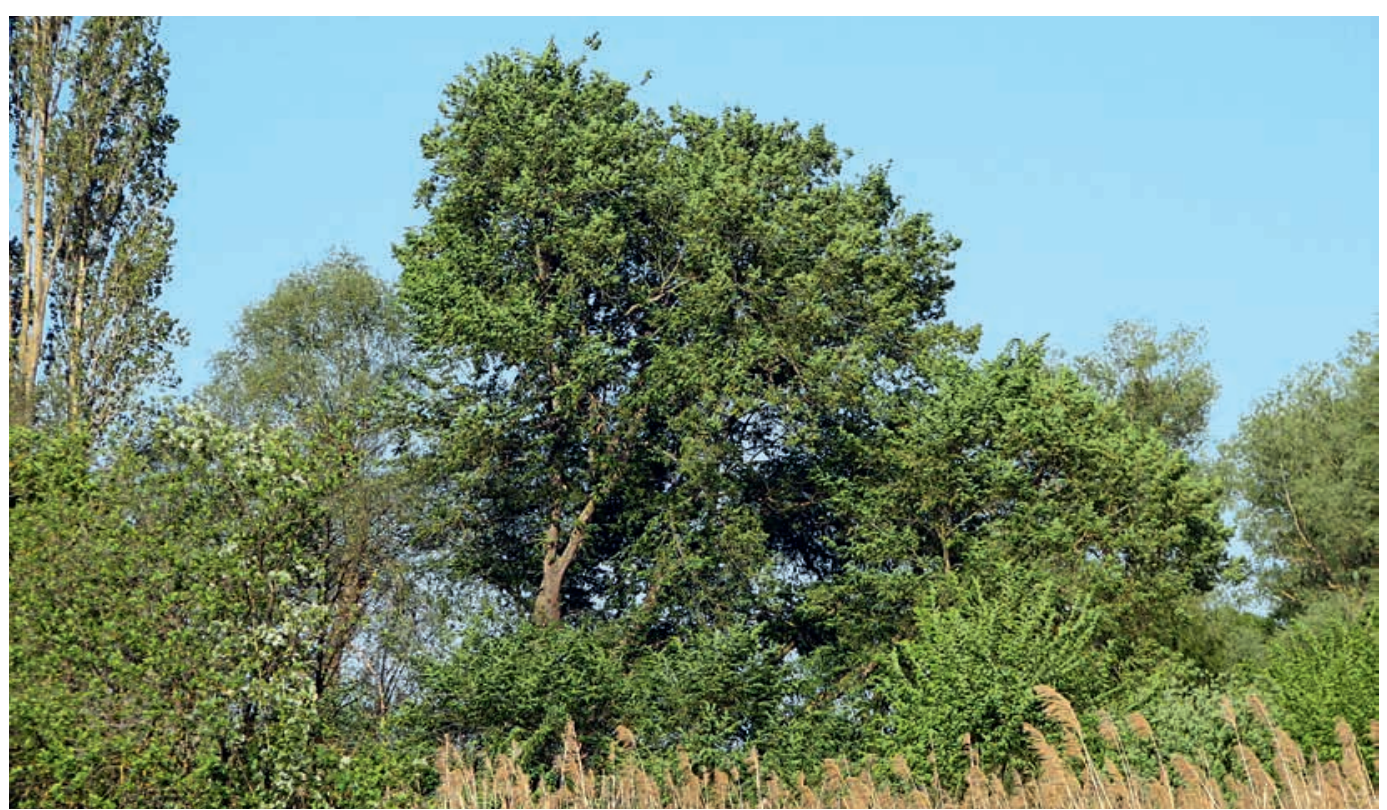

Abb. 1: Flatter-Ulme (Ulmus laevis) auf der Bodenseeinsel Reichenau. (Foto: V. M. DörkeN) 


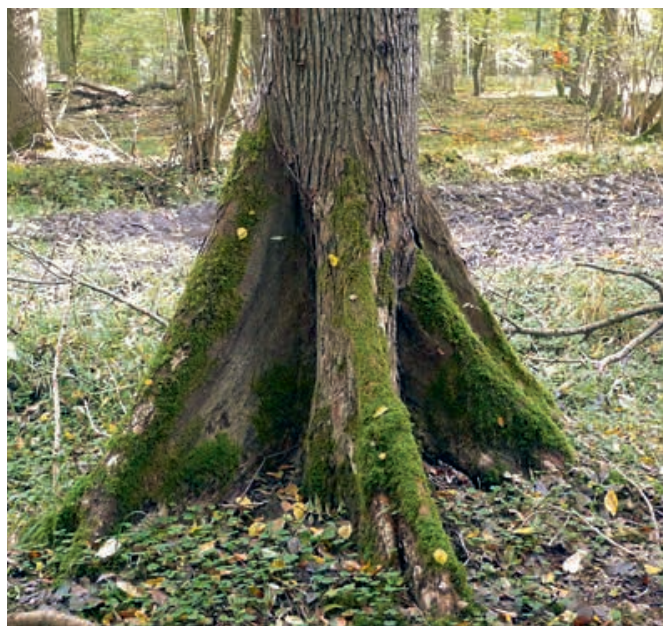

Abb. 2: Stamm einer Flatter-Ulme mit charakteristischen Brettwurzeln. (Foto: K. SIEPE)

falen beispielsweise wird dagegen die Flatter-Ulme offenbar gar nicht von der Krankheit beeinträchtigt. Trotzdem gehört Ulmus laevis zu den besonders stark gefährdeten heimischen Baumarten.

\section{Systematik}

Die Flatter-Ulme ist ein Ulmengewächs (Ulmaceae). Zu dieser Familie gehören auch die bei uns gelegentlich in Parkanlagen anzutreffenden Zelkovien (Zelkova) und Zürgelbäume (Celtis). Die Gattung Ulmus umfasst rund 30 meist winterkahle Arten. Ulmus laevis wird von Неув воек (1976) in die Sektion Blepharocarpus gestellt. Sie ist mit der Amerikanischen Ulme (Ulmus americana) aus der gleichen Sektion näher verwandt als mit den beiden anderen mitteleuropäischen Ulmen-Arten, der Berg-Ulme und der Feld-Ulme. Letztere sind in der Sektion Ulmus vereint. Mit diesen beiden Ulmen bildet die Flatter-Ulme keine Hybriden (Willner 1998).

\section{Name}

Die Flatter-Ulme wird auch Weiß-Rüster genannt, ältere Namen sind Wasser-Rüster und Glatt-Rüster (Schreiber 1957). Der Name Ulme wurde erst im späten Mittelalter von der lateinischen Bezeichnung des Baumes (ulmus) übernommen. Der zuvor verwendete Name Elm wurde allmählich verdrängt. Dieser lebt aber noch in einigen Ortsnamen wie z. B. Elmenhorst oder in der

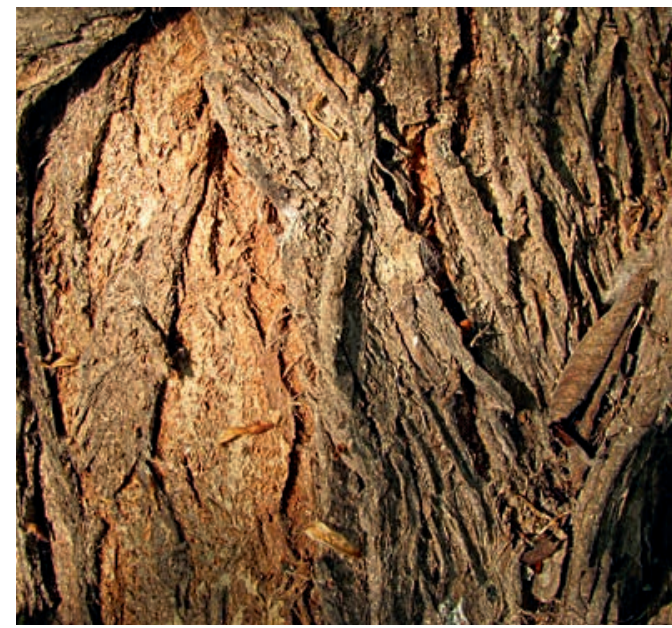

Abb. 3: Rauer Stamm einer Flatter-Ulme.

(Foto: V. M. Dörken)

Bezeichnung des häufig in Kreuzworträtseln abgefragten Höhenzuges bei Braunschweig (Elm) weiter (Düll \& Kutzelnigg 2016). Der Begriff Rüster wird vor allem für das Holz der Ulme verwendet. Die Vokabel ist zusammengesetzt aus dem mittelhochdeutschen rust (= Ulme) und der typischen Endung für Baumnamen (-ter, Schreiber 1957, Düll \& Kutzelnigg 2016). Der Artbeiname laevis bedeutet glatt und bezieht sich auf die glatte Blattoberfläche; andere Ulmen haben Blätter mit einer rauen Oberseite. Mit „Flatter“ wurde aufgegriffen, dass die lang gestielten Blüten und später entsprechend auch die Früchte im Gegensatz zu denen der anderen heimischen Ulmen im Wind flattern. Diese Eigenschaft kommt ebenso in älteren Synonymen wie U. effusa (flatternd) und $U$.pedunculata (mit deutlich gestielter Blüte) zum Ausdruck.

\section{Morphologie}

Die Flatter-Ulme wird an geeigneten Standorten bis $35 \mathrm{~m}$ hoch. Im Alter ist die Krone unregelmäßig rundlich, an besonders alten Exemplaren leicht hochgewölbt schirmartig mit vielen Wasserreisern. Am Stammfuß werden gelegentlich Brettwurzeln ausgebildet, ähnlich wie sie für viele tropische Bäume typisch sind. Unter den heimischen Bäumen ist dies ein einzigartiges Merkmal. Im Bereich der Stammbasis sind meist zahlreiche Schösslinge zu finden. Die Borke ist dunkelgrau, längsrissig und 


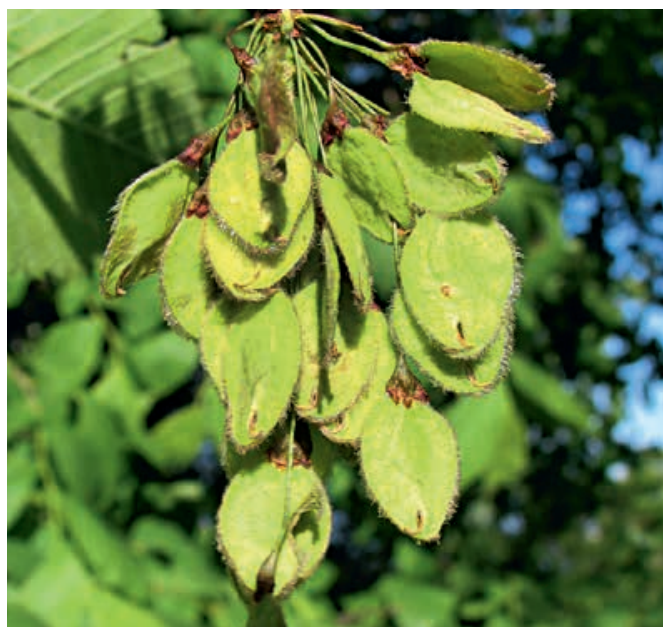

Abb. 4: Junger Fruchtstand einer Flatter-Ulme.

(Foto: V. M. Dörken)

blättert in kleinen, flachen, sich nach oben wölbenden Schuppen ab. Junge Triebe sind bräunlich bis graubraun und anfänglich weich behaart, verkahlen jedoch rasch und glänzen dann. Sie sind netzartig von flachen Leisten umspannt. Es fehlen aber deutliche Korkleisten, wie diese häufig bei der Feld-Ulme zu finden sind. Das Holz ist in einen breiten hellen, weißlich gelben Splintholzbereich und in einen dunkleren grau- bis bräunlich-gelben Kernholzbereich gegliedert. Dabei kann der Splintholzbereich bis zu zwei Drittel des Stammdurchmessers einnehmen (Sснӥтt et al. 2002).

Die Blätter stehen wie bei allen Ulmen wechselständig und sind kurz gestielt. Sie treiben meist etwa im April nach der Blütezeit aus. Die nicht runzelige Blattspreite hat einen extrem asymmetrischen Blattgrund. Die Blätter haben nur eine einzige Blattspitze, während die Blätter der Berg-Ulme häufig zwei kleinere Nebenspitzen aufweisen. Auf ihrer Oberseite sind die Blätter dunkelgrün, meist kahl oder nur leicht behaart und dadurch glänzend; die Unterseite ist deutlich weißlich und weich behaart und wirkt dadurch graugrün. Der Blattrand ist grob doppelt gesägt mit scharfen Zähnen. Die Spitzen der Blattzähne sind dabei stark einwärts gekrümmt. Nach einer gelben Herbstfärbung werden im Oktober die Blätter abgeworfen. Die dadurch entstehende Laubstreu ist leicht zersetzbar (SсHÜTT et al. 2002).

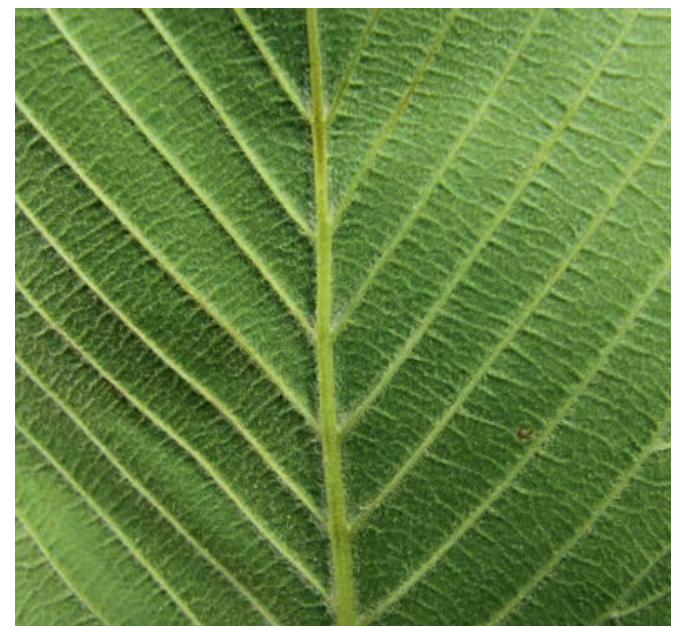

Abb. 5: Ausschnitt aus der Blattunterseite der Flatter-Ulme. (Foto: V. M. DöRKEN)

Die unauffällig grünlichen bis grünlich-violetten Blüten sind lang gestielt und erscheinen vor dem Blattaustrieb Ende März bis April. Im Vergleich zur Berg- und Feld-Ulme blüht die Flatter-Ulme etwa zwei Wochen später. Sie ist windbestäubt, gelegentlich kommt aber auch Insektenbestäubung vor (Kiermeier 1993b). Die Blüten stehen büschelartig beisammen und hängen weit über.

Wie bei allen Ulmen sind die Früchte geflügelte, einsamige Nüsschen, die durch den

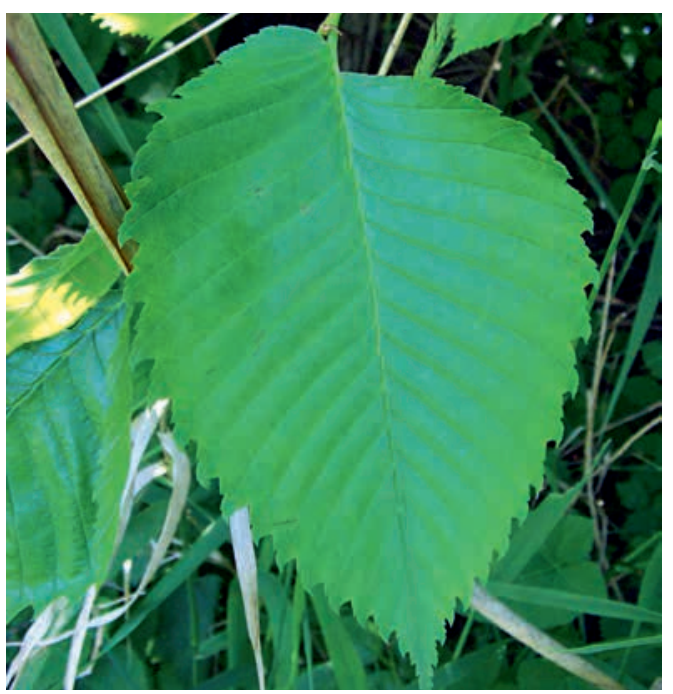

Abb. 6: Ganzes Blatt der Flatter-Ulme mit glatter Oberseite und schiefem Blattgrund. (Foto: V. M. DöRkeN) 


\begin{tabular}{|c|c|c|c|}
\hline Merkmal & U. glabra & $U$. minor & U. laevis \\
\hline Höhe & $30-40 \mathrm{~m}$ & $30-40 \mathrm{~m}$ & $20-30(-35) \mathrm{m}$ \\
\hline Krone & $\begin{array}{l}\text { unregelmäßig breit } \\
\text { ausladend }\end{array}$ & schmal, hochgewölbt & $\begin{array}{l}\text { unregelmäßig rund bis } \\
\text { schirmförmig }\end{array}$ \\
\hline Wasserreiser & selten & selten & zahlreich \\
\hline Borke & $\begin{array}{l}\text { lange glattbleibend, später } \\
\text { flach längsrissig }\end{array}$ & längsrissig bis gefeldert & $\begin{array}{l}\text { längsrissig, in flachen } \\
\text { Schuppen abblätternd }\end{array}$ \\
\hline Stamm & ohne Brettwurzeln & ohne Brettwurzeln & deutliche Brettwurzeln \\
\hline Wurzelbrut & fehlend & fehlend & zahlreich \\
\hline Triebe & rotbraun, behaart & $\begin{array}{l}\text { bräunlich bis grau-braun, } \\
\text { anfangs stark behaart und } \\
\text { drüsig }\end{array}$ & $\begin{array}{l}\text { bräunlich bis grau-braun, } \\
\text { anfänglich weich behaart, } \\
\text { rasch verkahlend, später } \\
\text { glänzend. }\end{array}$ \\
\hline Korkleisten & fehlend & deutlich & fehlend \\
\hline Blattlänge & $7-14 \mathrm{~cm}$ & $5-10 \mathrm{~cm}$ & $5-14 \mathrm{~cm}$ \\
\hline Blattstiel & $2-5 \mathrm{~mm}$ & $5-15 \mathrm{~mm}$ & $5-10 \mathrm{~mm}$ \\
\hline Blattspitze & 1- oder 3-spitzig & 1-spitzig & 1-spitzig \\
\hline $\begin{array}{l}\text { Blattspreiten- } \\
\text { basis }\end{array}$ & $\begin{array}{l}\text { stark asymmetrisch, die } \\
\text { längere Seite den Blattstiel } \\
\text { öhrchenartig überdeckend }\end{array}$ & $\begin{array}{l}\text { stark asymmetrisch, } \\
\text { ohne öhrchenartige } \\
\text { Ausbildungen }\end{array}$ & $\begin{array}{l}\text { stark asymmetrisch, } \\
\text { ohne öhrchenartige } \\
\text { Ausbildungen }\end{array}$ \\
\hline $\begin{array}{l}\text { Blatt- } \\
\text { behaarung }\end{array}$ & $\begin{array}{l}\text { beiderseits behaart, unter- } \\
\text { seits besonders auf den } \\
\text { Blattnerven, Haare steif }\end{array}$ & $\begin{array}{l}\text { oberseits verkahlend; } \\
\text { unterseits mit röt- } \\
\text { lich-braunen Achsel- } \\
\text { bärten, Haare weich }\end{array}$ & $\begin{array}{l}\text { oberseits verkahlend und } \\
\text { stumpf glänzend, unter- } \\
\text { seits dicht weiß behaart; } \\
\text { Haare weich }\end{array}$ \\
\hline $\begin{array}{l}\text { Blütezeit- } \\
\text { punkt }\end{array}$ & Ende März & Ende März & Ende März, April \\
\hline Blütenstiel & kurz & kurz & lang \\
\hline Perigon & $\begin{array}{l}\text { 4-5-teilig, braun } \\
\text { bewimpert }\end{array}$ & $\begin{array}{l}\text { 4-5-teilig, weiß } \\
\text { bewimpert }\end{array}$ & $\begin{array}{l}\text { 5-8-teilig, Rand leicht } \\
\text { ausgefranst }\end{array}$ \\
\hline Narbe & rosa bis rot & weiß & weiß \\
\hline Staubgefäße & $\begin{array}{l}\text { 5-6; deutlich die Blüten- } \\
\text { hülle überragend }\end{array}$ & $\begin{array}{l}\text { 4-5; deutlich die Blüten- } \\
\text { hülle überragend }\end{array}$ & $\begin{array}{l}\text { 5-8; nur wenig die } \\
\text { Blütenhülle überragend }\end{array}$ \\
\hline Fruchtlänge & $2-2,5 \mathrm{~cm}$ & $1,5-2 \mathrm{~cm}$ & bis $1 \mathrm{~cm}$ \\
\hline Fruchtflügel & kahl & kahl & stark bewimpert \\
\hline Same & zentral & im oberen Drittel & zentral \\
\hline
\end{tabular}

Tab. 1: Merkmale zur Unterscheidung der heimischen Ulmen-Arten. 
Wind ausgebreitet werden (Scheibenflieger). Bei der Flatter-Ulme sind die reifen Früchte wie auch schon die Blüten lang gestielt und hängen herab. Zur Reife ist der Fruchtstiel $5 \mathrm{~cm}$ lang. Die bis $1 \mathrm{~cm}$ breiten Früchte sind im Vergleich zur Bergund Feld-Ulme deutlich kleiner. Der häutige Flügelsaum, der den zentralen, den Samen umschließenden Bereich umgibt, ist deutlich schmaler und mit zahlreichen weißlichen Wimpernhaaren besetzt. Die Fruchtreife erfolgt recht früh bereits von Mai bis Juni. Bestimmungsmerkmale zur Unterscheidung der in Deutschland heimischen Ulmen-Arten sind in Tab. 1 zusammengestellt.

\section{Verbreitung und Vorkommen}

Die Flatter-Ulme ist eine ost- bis südosteuropäische Baumart, die bis in den westlichen Kaukasus verbreitet ist (Mitchell 1979). In Mitteleuropa gerät sie an ihre westliche Arealgrenze und wächst in Nord-, West- und Süddeutschland bevorzugt entlang der Stromtäler von Rhein, Elbe und Donau sowie deren Zuflüsse, in Ostdeutschland ist sie weiter verbreitet (KIERMEIER 1993b, floraweb.de). Die Flatter-Ulme ist ein typisches Element der Auen- und Ufergehölze, aber auch außerhalb des Überschwemmungsbereichs in Sumpf- und Bruchwäldern sowie in Niederungen zu finden (KIERMEIER 1993a, 1993b). Sie wächst in sommerwarmen Lagen auf sonnigen bis halbschattigen Standorten, bevorzugt nasse Bodenverhältnisse und stockt z. B. auf frischen, auch auf zeitweise überschwemmten, basenreichen, nährstoffreichen Lehm- und Tonböden. Die Flatter-Ulme ist kalkverträglich, mag aber eine schwach saure bis neutrale Bodenreaktion. Sie gilt weithin als Gleybodenzeiger, d. h. als Indikator für grundwasserbeeinflusste Böden (KIERMEIER 1993b, SсHÜтT et al. 2002). Pflanzensoziologisch betrachtet ist sie eine Charakterart des Traubenkirschen-Eschenwaldes (Pruno-Fraxinetum), tritt im Stieleichen-Ulmen-Auwald (Querco-Ulmetum) auf und ist Verbandscharakterart des Alno-Ulmion (Oberdorfer 1990). Bei Kartierungen der Flatter-Ulme ist es oft schwierig zu entscheiden, ob es sich tatsächlich um natürliche Vorkommen handelt oder ob es sich um gepflanzte oder aus Kultur verwilderte Individuen handelt (vgl. auch HaEupler et al. 2003).

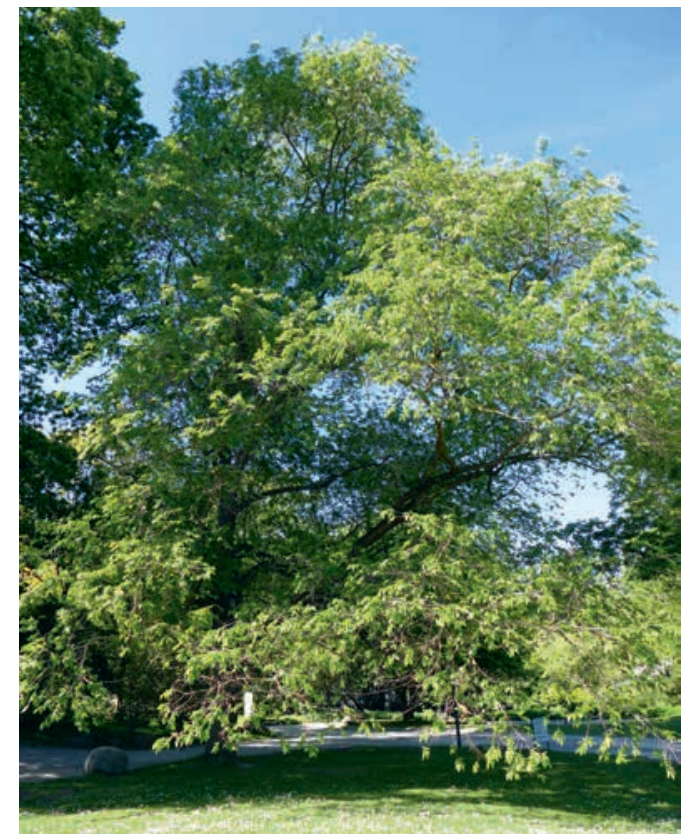

Abb. 7: Sapporo-Ulme (Ulmus 'Sapporo Autumn Gold') im Palmengarten. (Foto: H. Steinecke)

\section{Ulmensterben}

Die Bestände von Ulmen sind schon vor der Jahrtausendwende stark zurückgegangen. Einerseits ist dies auf Veränderung des Lebensraumes (Flussund Bachbegradigungen) zurückzuführen. Zum anderen hat aber auch das sogenannte Ulmensterben zum Rückgang der Populationen geführt.

Der erste Erreger des Ulmensterbens (die sogenannten Holländische Ulmenwelke), der Ascomycet (Schlauchpilz) Ophiostoma ulmi, wurde im 1. Weltkrieg aus Ostasien nach Frankreich eingeschleppt. Von dort hatte er sich rasant ausgebreitet. Die erste Welle des Ulmensterbens kam etwa um 1940 zum Erliegen. In den 1960er Jahren gab es eine neue Welle des Ulmensterbens. Diesmal war der Pilz Ophiostoma non-ulmi der Erreger. Dieser Pilz wird durch Ulmensplintkäfer (Scolytus scolytus, S. laevis und S. multistriatus) verbreitet (Sснмітт 2005). Ulmensplintkäfer sind Rüsselkäfer aus der Verwandtschaft der Borkenkäfer. Sie legen ihre Brutgänge, in denen sich die Larven entwickeln, in der Rinde der Wirtsbäume an. Ulmensplintkäfer werden deshalb als Rindenbrüter bezeichnet. Der Pilz besiedelt vor allem 


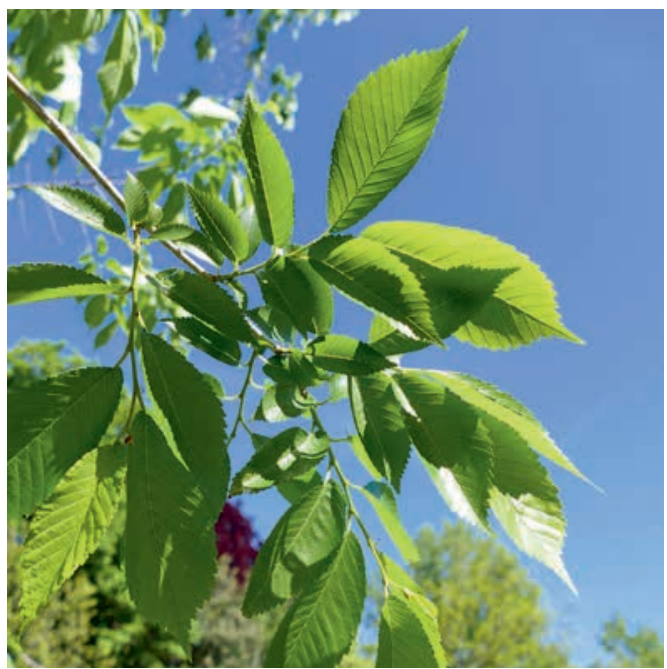

Abb. 8: Laub der Sapporo-Ulme. (Foto: H. STeinecke)

die Fraßgänge, die die Käfer im Holzkörper der jüngsten Jahresringe hinterlassen (DüLL \& KuTZELNigg 2016). Ausfliegende Jungkäfer kommen beim Verlassen des Baumes mit den Sporen in Kontakt, die an ihrem Körper hängen bleiben und somit ausgebreitet werden. Wenn die Tiere zu einem neuen Baum fliegen, können sie diesen neu infizieren. Die Infektion des Baumes mit dem Pilz erfolgt an jungen Trieben ( al. 2002). Der Pilz wird über den Holzkörper des Baumes in andere Kronenbereiche verteilt. In der Wirtspflanze scheidet der Pilz für den Wirt toxische Substanzen aus, die zum Kollabieren von benachbarten Gewebezellen führen. Eine Folge ist die Verstopfung der Leitungsbahnen des Xylems (Tracheen) und damit eine Unterbrechung des Wassertransportes. Dadurch welken Blätter und kleinere Seitenäste, was zu einem frühzeitigen Blattabwurf und Eintrocknen anfänglich kleinerer Seitentriebe und später ganzer Astpartien führt. Die Verstopfung des Xylems lässt sich im jüngsten Jahresring an kleinen braunschwarzen Punkten auch mit bloßem Auge erkennen (SсHÜтt et al. 2002). Der Befall kann sich innerhalb eines Jahres auf die gesamte Krone ausdehnen. Im Gegensatz zur Feld- und Berg-Ulme ist die Flatter-Ulme weitaus seltener vom Ulmensterben betroffen. Forstwissenschaftler führen dies darauf zurück, dass die Art für Ulmensplintkäfer als Futterpflanze weniger attraktiv ist (Preller 2010).
Die Bekämpfung des Ulmensterbens ist recht schwierig und bedarf einerseits der Bekämpfung des Überträgers, also des Ulmensplintkäfers. Die kann durch Pflanzung von „Fangbäumen“ erfolgen. Eine andere Möglichkeit ist der Einsatz von Insektiziden und Fungiziden gegen den Pilz (Schütт et al. 2002). Da es sich bei den heimischen Ulmen um Arten der Auen- und Flusslandschaften handelt, ist chemischer Pflanzenschutz der Bäume kaum möglich, ohne eine Belastung der Gewässer auszuschließen. Besonders Bestände mit einer hohen Individuendichte in Kombination mit Grundwasserabsenkungen am Standort führen zu einem vermehrten Befall (Düll \& KutZelnigG 2016).

Bei dem Ulmensterben des 20. Jahrhunderts handelt es sich nicht um den ersten Rückgang von Ulmen in Mitteleuropa seit dem Ende der letzten Eiszeit. Ulmen waren in der Klimastufe der Mittleren Warmzeit (etwa 8000-4000 v. Chr.) weitaus häufiger anzutreffen als heute. Sie bildeten Mischwälder zusammen mit Eichen und Linden. Für das 4. Jahrtausend vor der Zeitenwende zeigt sich in den Pollendiagrammen aus Mittel- und Nordwesteuropa ein markanter Rückgang des Ulmenpollens. Dieser erfolgte mitunter sehr abrupt innerhalb von Jahren oder Jahrzehnten, jedoch nicht überall gleichzeitig. Die Gründe für diesen Rückgang des Ulmenpollens sind bis heute nicht abschließend geklärt. Neben Klimaveränderungen und einer Verschlechterung der Böden werden anthropogene Ursachen wie Rodung und Schwächung der Bäume sowie verminderte Blüte durch Schneiteln zur Laubheugewinnung angenommen. Daneben liegen auch für die damalige Zeit starke Indizien für eine Schädigung der Bäume durch eine vom Ulmensplintkäfer übertragene Pilzerkrankung vor. Vermutlich handelte es sich um eine Kombination und wechselseitige Beeinflussung dieser Ursachen (Garbett 1981, Girling \& Greig 1985, Perry \& Moore 1987, Graumlich 1993, Parker et al. 2002).

Trotz allem sind Ulmen sehr schöne Gehölze, die unsere Gärten bereichern können. Im Idealfall werden solche Ulmen verwendet (z. B. Arten aus Asien), die gegen das Ulmensterben resistent 
sind. Im Palmengarten steht eine attraktive und mittlerweile recht große Sapporo-Ulme seitlich der Galerie West auf dem Weg in Richtung Großer Weiher. 'Sapporo Autumn Gold' ist eine in Amerika gezüchtete Kreuzung der asiatischen Arten $U$. davidiana var. japonica und $U$. pumila. Im Jahr 1958 wurden an der University von Wisconsin aus Saatgut des Hokkaido University Botanical Gardens, Sapporo, abertausende Sämlinge angezogen, mit dem Erreger infiziert und über Jahre selektiert. 1975 wurde die Sorte patentiert. Die Sapporo-Ulme im Palmengarten wurde 1980 als junger Baum aus Amerika eingeführt und im Palmengarten gepflanzt. Die Basis der Blattspreite ist etwas schief, ein typisches Merkmal für Ulmen-Blätter. Im Herbst färbt sich die Sapporo-Ulme leuchtend gelb.

Im Botanischen Garten Frankfurt gibt es drei junge Exemplare der Flatter-Ulme, dem eigentlichen Baum des Jahres 2019. Der Botanische Garten erhielt sie im Jahr 2003 aus Wolfen in Sachsen-Anhalt. Ein Exemplar steht untypisch am Heidehang, einem relativ trockenen Standort auf sandig-humosem Boden. Die beiden anderen stehen auf einem betont bodenfrischen Lehmboden in der Nähe des Bachlaufes am Rand des Eichen-Hainbuchenwaldes.

\section{Dank}

Für den Hinweis auf das „erste Ulmensterben“ bedanken wir uns herzlich bei Dr. Till Kasielke (Mülheim/Ruhr).

\section{Literatur}

Düll, R. \& Kutzelnigg, H. 2016: Taschenlexikon der Pflanzen Deutschlands und angrenzender Länder, 8. Aufl. Wiebelsheim.

Garbett, G. G. 1981: The elm decline: the depletion of a resource. - New Phytologist 88: 573-585.

Girling, M. A. \& Greig, J. 1985: A first fossil record for Scolytus scolytus (F.) (elm bark beetle): its occurrence in elm decline deposits from London and the implications for neolithic elm disease. - J. Archaeolog. Sci. 12: 347-351.

Graumlich, L. J. 1993: High resolution pollen analysis provides new perspective on catastrophic elm decline. - Trends Ecol. \& Evol. 8: 387-388.
Haeupler, H., Jagel, A. \& Schumacher, W. 2003: Verbreitungsatlas der Farn- und Blütenpflanzen in Nordrhein-Westfalen - Landesanstalt für Ökologie, Bodenordnung und Forsten Nordrhein-Westfalen. Hrsg. LÖBF. - Recklinghausen.

Неуввоек, H. M. 1976: Sistematiek en nomenklatuur van het geslacht Ulmus. - Medded. Rijksinstit.ondersoek bos en landschaps. De Dorschkamp. Nr. 6. Wageningen.

Kasielke, T. 2014: Spätquartäre Landschaftsentwicklung im oberen Emscherland. - Diss., Geograph. Inst., Ruhr-Universität Bochum.

Kasielke, T. 2016: Nacheiszeitliche Waldentwicklung in Westfalen. - In: Grothues, R., Otto, K.-H. \& Wieneke, M. (Hrsg.): Westfalen Regional 3 (= Siedlung und Landschaft in Westfalen 41):: 44-45.

Kiermeier, P. 1993a: Lebensbereiche der Gehölze eingeteilt nach dem Kennziffersystem. Fördergesellschaft „Grün ist Leben". - Pinneberg.

Kiermeier, P. 1993b: Wildgehölze des mitteleuropäischen Raumes, BdB Handbuch Teil VIII. Fördergesellschaft „Grün ist Leben“. - Pinneberg.

Mitchell, A. 1979: Die Wald- und Parkbäume Europas, 2. Aufl. - Berlin.

Oberdorfer, E. 1990: Pflanzensoziologische Exkursionsflora, 6. Aufl. - Stuttgart.

Parker, A. G., Goudie, A. S., Anderson, D. E., Robinson, M. A. \& Bonsall, C. 2002: A review of the mid-Holocene elm decline in the British Isles. - Prog. Physic. Geogr. 26: $1-45$.

Perry, I. \& Moore, P. D. 1987: Dutch elm disease as an analogue of Neolithic elm decline. - Nature 326: 72-73.

Pотт, R. 1995: Die Pflanzengesellschaften Deutschlands, 2. Aufl. - Stuttgart.

Preller, J. 2010: Das heimliche Sterben der Ulmen. - Landwirtschaftl. Wochenbl. 2010: 52-53.

Raabe, U., Büscher, D., Fasel, P., Foerster, E., Götte, R., Haeupler, H., Jagel, A., Kaplan, K., Keil, P., Kulbrock, P., Loos, G. H., Neikes, N., Schumacher, W., Sumser, H. \& Vanberg, C. 2011: Rote Liste und Artenverzeichnis der Farn- und Blütenpflanzen, Pteridophyta et Spermatophyta, in Nordrhein-Westfalen, 4. Fassg. LANUV-Fachber. 36: 51-183.

Sснӥтt, P., Sснuск, H. J. \& Sтімм, B. 2002: Lexikon der Baum- und Straucharten. - Hamburg.

Willner, W. 1998: Neue Befunde an Ulmus in Österreich. Fl. Austr. Novit. 5: 26-33.

\section{Anschriften der Autoren}

Dr. Veit Martin Dörken, Universität Konstanz, Fachbereich Biologie M 613, Universitätsstraße 10, 78457 Konstanz, E-Mail: veit.doerken@uni-konstanz.de

Dr. Armin Jagel, Danziger Straße 2,44789 Bochum, E-Mail: armin@jagel.nrw 